\title{
EpCAM expression in esophageal cancer and its correlation with immunotherapy of solitomab
}

\author{
Lan Yu ${ }^{1}$, Qing-Ming Guo ${ }^{2}$, Yu Wang ${ }^{1}$, Yan Xu ${ }^{1}$, Li Liu $^{1}$, Xiao-Tao Zhang ${ }^{1} \wedge$ \\ ${ }^{1}$ Department of Stereotactic Radiotherapy, Affiliated Qingdao Central Hospital, Qingdao University, Qingdao, China; ${ }^{2}$ Biotherapy Center, Affiliated \\ Qingdao Central Hospital, Qingdao University, Qingdao, China \\ Contributions: (I) Conception and design: L Yu; (II) Administrative support: XT Zhang; (III) Provision of study materials or patients: QM Guo; (IV) \\ Collection and assembly of data: Y Wang, Y Xu; (V) Data analysis and interpretation: L Yu, L Liu; (VI) Manuscript writing: All authors; (VII) Final \\ approval of manuscript: All authors. \\ Correspondence to: Xiao-Tao Zhang. Department of Stereotactic Radiotherapy, Affiliated Qingdao Central Hospital, Qingdao University, No. 127, \\ Siliu South Road, Shibei District, Qingdao 266042, China. Email: 18669710019@126.com.
}

Background: Recurrence of esophageal cancer (EC) after chemotherapy may mainly be explained by the existence of chemotherapy-resistant cells, and an effective drug against chemotherapy-resistant cells is highly sought. The aim of this study was to investigate the cytotoxicity of bispecific antibody solitomab combined with $\gamma \delta$ T cells on Eca109 cell spheres.

Methods: We cultured Eca109 cell spheres in serum-free medium, and the morphological differences between wild-type Eca109 cells and Eca109 cell spheres were compared by microscope and flow cytometry. Different concentrations of nanoparticle albumin-bound paclitaxel (Nab-PTX) and cisplatin were used to treat the two groups of cells and compare their drug resistance. Flow cytometry was then used to detect the expression level of epithelial cell adhesion molecule (EpCAM) and the cytotoxicity of $\gamma \delta \mathrm{T}$ cells combined with bispecific antibody solitomab on the two groups.

Results: Flow cytometry analysis showed that Eca109 cell spheres were smaller in size and had less cytoplasmic granules and CCK-8 assay showed that the viability of Eca109 cell spheres treated with different concentrations of Nab-PTX and cisplatin was significantly higher than that of wild-type Eca109 cells $(\mathrm{P}<0.05)$. Flow cytometry also showed that the expression level of EpCAM on Eca109 cell spheres was higher than that of wild-type Eca109 cells. Co-culture experiment showed that there was no significant difference in the cytotoxicity of $\gamma \delta$ T cells to wild-type Eca109 cells and Eca109 cell spheres without solitomab. However, after adding solitomab, the cytotoxicity of $\gamma \delta$ T cells to Eca109 cell spheres was significantly higher than that of wild-type Eca109 cells $(\mathrm{P}<0.05)$.

Conclusions: EC Eca109 cell spheres have strong stem cell characteristics such as multidrug resistance and may contain a high proportion of EC stem cells. Further, EC Eca109 cell spheres have a high expression level of EpCAM, and EpCAM may be one of the markers of EC stem cells. Therefore, EpCAM could be used as a potential molecular target of immunotherapy for EC, and solitomab may become an effective immunotherapeutic drug for chemotherapy-resistant EC cells.

Keywords: Esophageal cancer (EC); cancer stem cell (CSC); epithelial cell adhesion molecule (EpCAM); solitomab

Submitted Jan 12, 2021. Accepted for publication Apr 21, 2021.

doi: $10.21037 /$ jtd-21-442

View this article at: http://dx.doi.org/10.21037/jtd-21-442

^ ORCID: Lan Yu, 0000-0003-0448-1887; Xiao-Tao Zhang, 0000-0001-7213-4970. 


\section{Introduction}

Esophageal cancer (EC) is a devastating malignancy and carries the seventh highest mortality and sixth highest morbidity of malignant tumors worldwide (1). Nearly two-thirds of EC patients had locally advanced stage or distant metastasis at the time of diagnosis, by which time they had lost the opportunity for surgery (2) and systemic chemotherapy was the only remaining option. However, drug resistance and tumor progression occurs soon after systemic chemotherapy, and the overall 5 -year overall survival (OS) rate of these patients remains at $15-25 \%$ (3). Studies have reported that EC stem cells were mainly responsible for radiotherapy and chemotherapy resistance and treatment failure $(4,5)$. These cancer stem cells (CSCs) harbor unique properties including self-renewal, high tumorigenicity, proliferation, migration, and resistance to conventional therapy, leading to treatment failure (6). Therefore, finding effective drugs to eliminate these chemotherapy-resistant cells is likely to improve the survival rate in EC patients.

Serum-free suspension culture is to add specific growth factors to the serum-free medium, which can make most cancer cells stop growing and eventually die, while cancer stem cells can continue to proliferate to form suspended cell spheres. In 2003, Singh and Hemmati et al. $(7,8)$ successfully cultured brain tumor cell spheres from glioma and medulloblastoma cells by serum-free suspension culture. These cell spheres were confirmed to be tumor stem cells and expressed tumor stem cell markers. Wang et al. successfully obtained passaging cell spheres from KYSE150 and TE1 EC cell lines by serum-free suspension culture. They found these sphere cells possessed cancer stem cell characteristics such as high invasion, self-renewal and resistance to radiation.

Epithelial cell adhesion molecule (EpCAM) is a type I transmembrane glycoprotein antigen, which is expressed in a variety of epithelial tumors (9-11) and some CSCs, and is considered to be an important biomarker of some malignant tumors $(12,13)$. EpCAM has become an effective marker for the identification and isolation of some CSCs and one of the targets of cancer immunotherapy (14). However, its expression in EC stem cells remains unclear.

Solitomab is an EpCAM/CD3 bispecific single-chain antibody construct which can bind to tumor cells expressing EpCAM and T cells simultaneously to activate $T$ cells to kill tumor cells (15). Its anti-tumor mechanism is to activate $\mathrm{T}$ cells to release granzyme and perforin, which dissolve tumor cells and induce apoptosis (16). Studies have confirmed that solitomab can suppress uterine sarcoma, colon cancer, and pancreatic cancer cell proliferation $(15,17,18)$. However, the effect of solitomab on EC cells has not been reported.

$\gamma \delta \mathrm{T}$ cells are a population of non-specific killer T cells containing a T cell receptor (TCR) made up of $\gamma$ and $\delta$ chains. Unlike $\alpha \beta$ T cells, $\gamma \delta$ T cells recognize their peptidic antigens in a major histocompatibility complex (MHC)independent manner (19). $\gamma \delta \mathrm{T}$ cells can also express $\mathrm{CD} 3$, which can be effectively combined with solitomab. Therefore, in this study, we selected $\gamma \delta \mathrm{T}$ cells as effector cells and investigated the cytotoxicity of bispecific antibody solitomab combined with $\gamma \delta$ T cells on EC cell spheres, to explore an effective drug against chemotherapy-resistant cells.

We present the following article in accordance with the MDAR reporting checklist (available at http://dx.doi. org/10.21037/jtd-21-442).

\section{Methods}

\section{EC Eca109 cells culture}

A Human EC Eca109 cell line (RRID: CVCL_6898) was donated by the Biotherapy Center of Qingdao Central Hospital. The cryopreserved cells were removed from the liquid nitrogen tank at $-80{ }^{\circ} \mathrm{C}$ and then resuscitated. The cells were cultured in fresh Roswell Park Memorial Institute (RPMI) 1640 (HyClone, USA) containing 10\% fetal bovine serum (FBS) (Gibco, USA) at $37{ }^{\circ} \mathrm{C}$ with $5 \% \mathrm{CO}_{2}$ and the culture medium was changed every 2 days.

\section{Eca109 cell spheres culture}

When the growth density of Eca109 cells in the medium reached $80 \%$ or more, the cells were digested with $0.25 \%$ Trypsin containing $0.02 \%$ EDTA (Gibco, USA) for 3 minutes. The cells were cultured in serum-free RPMI1640 medium and B27 Supplement (Huiying Biotechnology Co., Ltd. Shanghai, China) at $37{ }^{\circ} \mathrm{C}$ with $5 \% \mathrm{CO}_{2}$. On the third day of culture, cell spheres with regular shape, small size, and suspension growth appeared. After culturing for 7-8 days, the cells were blown regularly, and the suspended cell spheres were collected for the experiment.

\section{Cell chemotherapy resistance experiment}

The Eca109 cell spheres were blown into a single cell 
suspension and centrifuged at 1,000 rpm for $5 \mathrm{~min}$. Supernatant was then discarded, and the cell concentration was adjusted to $5 \times 10^{5} / \mathrm{mL}$ with RPMI 1640 medium of $10 \%$ FBS. The single cell suspension was inoculated into a 96well plate with $5 \times 10^{4}$ cells per well, then placed at $37^{\circ} \mathrm{C}$, with $5 \% \mathrm{CO}_{2}$. On the following day, the cells were treated with different concentrations of Nab-PTX $(0,0.1,0.4,1$, and $2 \mu \mathrm{g} / \mathrm{mL})$ and cisplatin $(0,3,10,30$, and $50 \mu \mathrm{g} / \mathrm{mL})$ for 48 hours and a $10 \mu \mathrm{L}$ CCK-8 solution was then added to culture the cells for 3 hours. The OD value of cells in each well was detected by Elisa at $490 \mathrm{~nm}$, and the viability rate of cells was calculated. The cell viability rate $\%=$ (experimental group OD - blank group OD)/(control group OD - blank group OD) $\times 100 \%$. The histograms of the cell viability of wild-type Eca109 cells and Eca109 cell sphere cells treated with Nab-PTX and cisplatin respectively were then drawn.

\section{Analysis of EpCAM expression level}

The wild-type Eca109 cells and Eca109 cell sphere cells were collected and washed with ice-cold PBS, and $100 \mu \mathrm{L}$ of cell suspension was then made. The cell suspension was mixed with a PE-labeled anti-EpCAM monoclonal antibody (BioLegend, United States), or a corresponding isotype control antibody, and then incubated at room temperature for $20 \mathrm{~min}$. After incubation, the cells were washed with PBS again and analyzed by BD Calibur flow cytometry. The expression level of EpCAM was then analyzed by Cell quest software.

\section{Expansion and culture of $\gamma \delta$ T cells in vitro}

Human peripheral blood samples were obtained from healthy volunteer blood donors who had previously their written informed consent. Human peripheral blood (50ml) was collected and centrifuged and the upper plasma was retained for cell culture. Peripheral blood mononuclear cells (PBMC) were isolated and extracted by the lymphocyte separation solution Ficol (GE Healthcare, Uppsala, Sweden). Those with a cell density of $2 \times 10^{6} / \mathrm{mL}$ were cultured in RPMI 1640 medium containing $5 \%$ autologous plasma and Interleukin-2 (IL-2) (Quangang Pharmaceutical Co., Ltd. Shandong, China) and zoledronic acid (Zhengda Tianqing Pharmaceutical Group Co., Ltd. China) were added to the culture system with a final concentration of $500 \mathrm{U} / \mathrm{mL}$ (IL-2) and $1 \mu \mathrm{M}$ (zoledronic acid), respectively. Thereafter, an appropriate amount of RPMI1640 medium containing IL-2 (concentration of $200 \mathrm{U} / \mathrm{mL}$ ) and $5 \%$ autologous plasma were added to the culture system according to the specific conditions.

\section{Sorting of $\gamma \delta$ T cells}

The $\gamma \delta \mathrm{T}$ cells in culture system were collected and incubated with anti-human TCR V $\delta$ 2-biotin (Miltenyi, Germany) and anti-biotin MicroBeads (Miltenyi, Germany) at $4{ }^{\circ} \mathrm{C}$ for 15 minutes. Cells were washed twice with PBS to remove the non-specific binding antibodies and magnetic beads. After resuspension, $\gamma \delta \mathrm{T}$ cells were sorted by VarioMACS sorting column according to the instructions and flow cytometry then analyzed that the positive rate of sorted cells was more than $90 \%$.

\section{Co-culture of $\gamma \delta$ T cells with EC cells}

$\gamma \delta \mathrm{T}$ cells cultured for 14 days were co-cultured with wildtype Eca109 cells or Eca109 cell sphere cells at an effectortarget ratio of 1:1 (the number of effector cells and target cells was $2 \times 10^{5}$ ) in a 24 -well plate. Solitomab (Amgen, United States) was added to the experimental group (the final concentration was $30 \mathrm{ng} / \mathrm{mL}$ ), while solitomab was not added to the control group. Separate culture holes of wildtype Eca109 cells and Eca109 cell sphere cells were then set up to determine the background death level. After 12 hours of culture, all cells in the co-culture system were collected and analyzed by flow cytometry.

\section{Cytotoxicity assay}

$\gamma \delta \mathrm{T}$ cells were used as effector cells while CFSE (Invitrogen, Carlsbad, USA) labeled wild type Eca109 cells or Eca109 cell sphere cells were used as target cells. The specific steps of CFSE labeled wild-type Eca109 cells or Eca109 cell sphere cells before co-culture were as follows: Cells were re-suspended in PBS containing 1\% BSA with a final cell concentration of $5 \times 10^{6} / \mathrm{mL}$. The cell suspension was added with the same volume of CFSE working solution $(2 \mu \mathrm{M})$, incubated at $37^{\circ} \mathrm{C}$ for $10 \mathrm{~min}$, then added with five times the volume of complete medium and placed on ice for $5 \mathrm{~min}$ to terminate the staining reaction. The cells were washed with PBS containing 1\% BSA three times, then resuscitated with complete culture medium for follow-up experiments. After 12 hours of co-culture, all cells in the co-culture group and single culture group were collected into tubes and washed twice with PBS then re-suspended in $200 \mu \mathrm{L}$ PBS. Each tube 

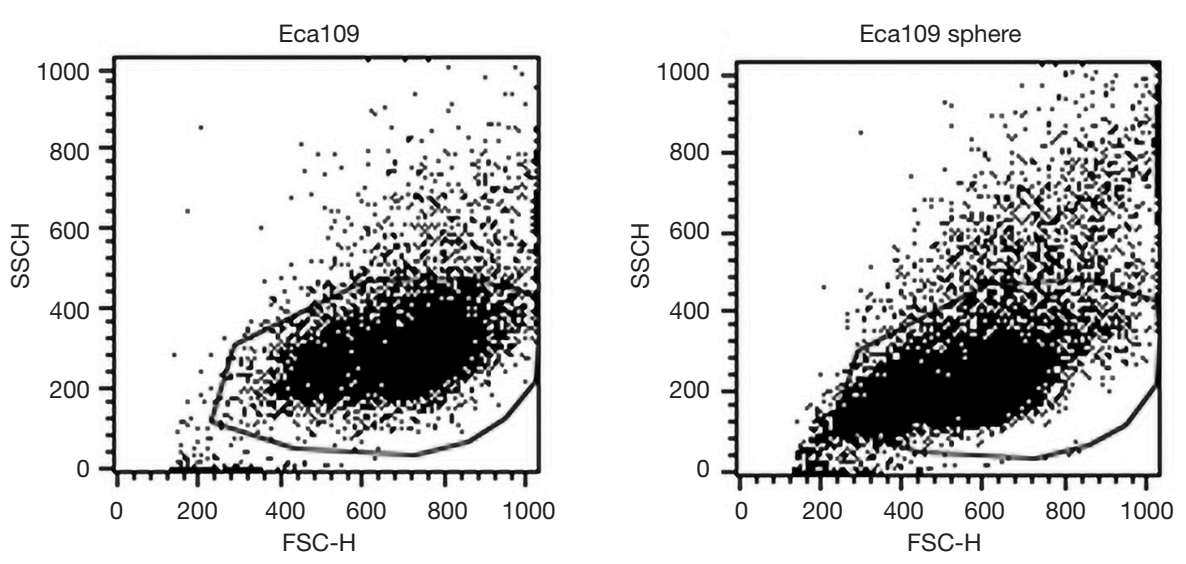

Figure 1 Morphology of wild-type Eca109 cells and Eca109 cell sphere by flow cytometry. SSCH, side scatter height; FSC-H, forward scatter-height.

was added with $20 \mu \mathrm{L}$ PI $(1 \mu \mathrm{g} / \mathrm{mL})$ and then was placed on ice for $10 \mathrm{~min}$. The reaction was terminated by adding 200 $\mu \mathrm{L}$ of complete medium and the cells were detected by FACS Calibur. The CFSE ${ }^{\text {high }}$ PI-cells detected by flow cytometry were survivable Eca109 cells or Eca109 cell sphere cells and the calculation of cytotoxicity was based on the degree of reduction of viable target cells with the ability to retain CFSE and exclude PI ( $\left.\mathrm{CFSE}^{\text {high }} \mathrm{PI}^{-}\right)$.

\section{Statistical method}

SPSS 23.0 (SPSS, IBM, Chicago, USA) statistical software was used for statistical analysis. The data were expressed by mean \pm standard deviation (SD), and the mean among samples were compared by t-test. The difference was statistically significant at $(\mathrm{P}<0.05)$.

\section{Results}

\section{Morphology of EC Eca109 sphere cells}

Under high-power microscope, EC Eca109 cells were irregular round or fusiform, with abundant cytoplasm, round nucleus, compact arrangement between cells, and adherent growth. On the third day of culture, small and regular suspension-growing cell spheres formed in serumfree medium and the first generation of cell spheres with small size, round shape, and tight connection between cells were obtained on the 12th day of culture. The cells were then collected and passaged, and the second generation of cell spheres with small size, round shape, less cytoplasm, and large nucleus were formed on the 8th day of culture. Compared with the first generation of cell spheres, the second generation formed faster and were smaller in size and of a more regular in shape.

Flow cytometry analysis showed that the forward scatter (FSC) and side scatter (SSC) values of Eca109 cell spheres were lower than those of wild-type Eca109 cells, suggesting that Eca109 cell spheres were smaller in size and had less cytoplasmic granules (Figure 1).

\section{Drug resistance experiment of Eca109 cells sphere cells}

After the wild-type Eca109 cells and Eca109 cell sphere cells were treated with different concentrations of NabPTX and cisplatin for 24 hours, the growth of both groups of cells cultured in the 96-well plate was inhibited. With increasing drug concentration, the proportion of surviving cells decreased gradually, the cell morphology changed, and the cell membranes were shrunken and incomplete.

CCK-8 assay showed that the cell viability rate of Eca109 cell spheres was significantly higher than that of wild-type Eca109 cells. This suggests that Eca109 cell spheres have stronger drug resistance than wild-type Eca109 cells and may contain a higher proportion of EC stem cells (Figures 2,3).

\section{EpCAM expression of Eca109 cells sphere cells}

The above research shows that Eca109 cell spheres may contain a higher proportion of EC stem cells. Many studies have reported that the frequency and range of EpCAM expression in some CSCs were more extensive and frequent 

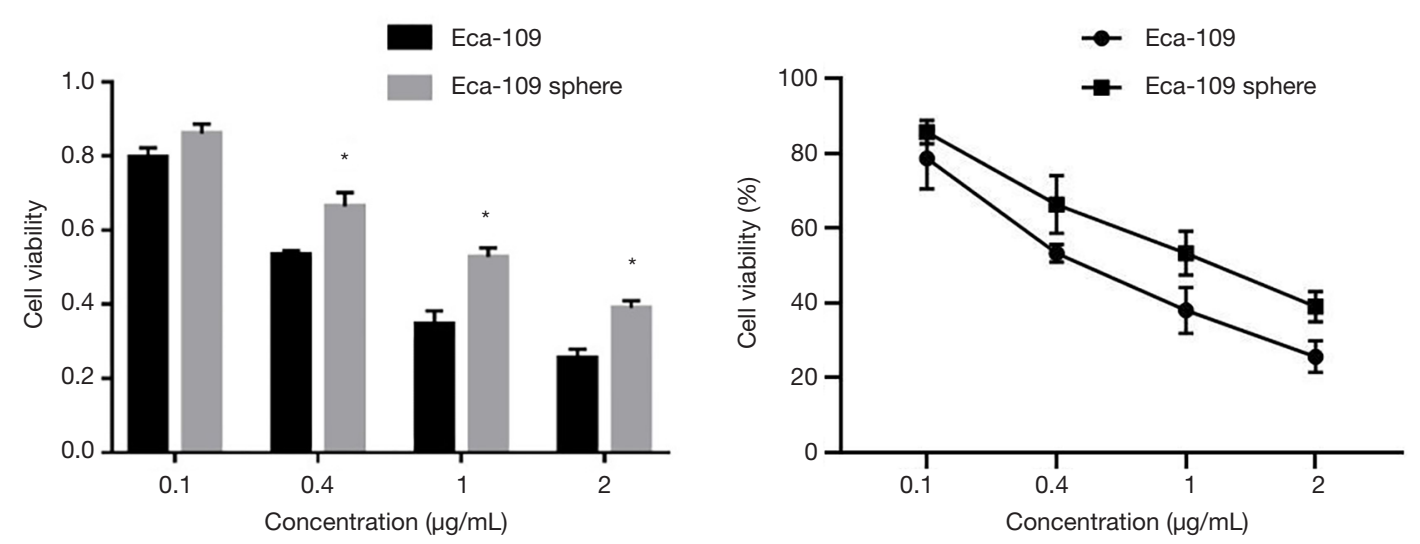

Figure 2 Cell viability of wild-type Eca109 cells and Eca109 cell sphere cells treated with Nab-PTX. *, with the increase of Nab-PTX concentration, cell viability rate of wild-type Eca109 cells and Eca109 cell sphere cells decreased, while the cell viability rate of Eca109 cell spheres was significantly higher than that of wild-type Eca109 cells $(\mathrm{P}<0.05)$.
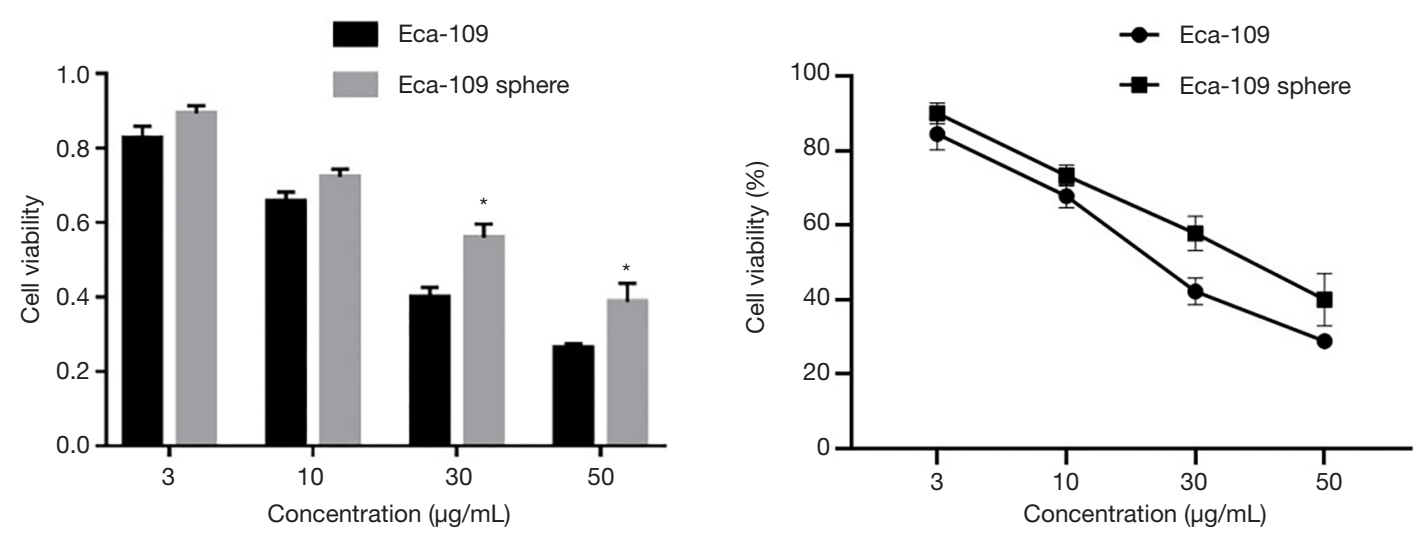

Figure 3 Cell viability of wild-type Eca109 cells and Eca109 cell sphere cells treated with cisplatin. *, with the increase of cisplatin concentration, Cell viability rate of wild-type Eca109 cells and Eca109 cell sphere cells decreased, while the cell viability rate of Eca109 cell spheres was significantly higher than that of wild-type Eca109 cells $(\mathrm{P}<0.05)$.

than non-CSCs $(14,20,21)$. Flow cytometry showed that EpCAM expression was also observed in wild type Eca109 cells and Eca109 cell sphere cells, but the expression level on Eca109 cell sphere cells was higher than that of wildtype Eca109 cells (Figure 4).

\section{Cytotoxicity of Solitomab combined with $\gamma \delta$ T cells to Eca109 cell sphere cells}

Cultured $\gamma \delta \mathrm{T}$ cells (effector cells) were co-cultured with wild-type Eca109 cells (target cells) or Eca109 cell sphere cells (target cells) at the effector-target ratio of $1: 1$ for 10 to 12 hours. Co-culture experiment showed that there was no significant difference in the cytotoxicity of $\gamma \delta$ T cells to wild type Eca109 cells and Eca109 cell sphere cells without solitomab. However, after adding solitomab, $\gamma \delta \mathrm{T}$ cells significantly enhanced the cytotoxicity of both groups of cells and the cytotoxicity of $\gamma \delta$ T cells to Eca109 cell sphere cells was significantly higher than that of wild type Eca109 cells $(\mathrm{P}<0.05)$ (Figure 5).

\section{Discussion}

EC is one of the most common fatal cancers worldwide with an overall 5 -year OS ranging from $15 \%$ to $25 \%$ (3). Despite advances in surgical therapy for ESCC, local recurrence and distant metastasis are the main reasons for postoperative treatment failure. Nearly two-thirds of EC 


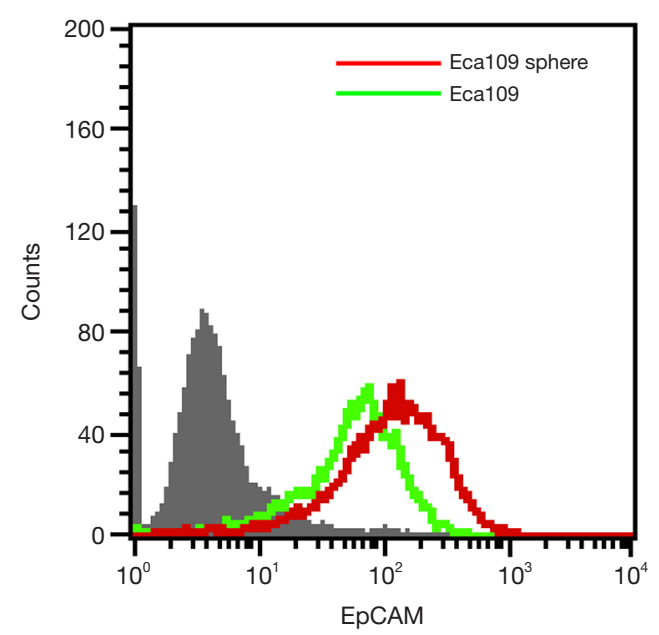

Figure 4 Analysis of EpCAM expression level in wild-type Eca109 cells and Eca109 cell sphere cells by flow cytometry. EpCAM, epithelial cell adhesion molecule.

patients had locally advanced stage or disseminated disease at diagnosis and had lost the opportunity for surgery (2). In these patients, systemic chemotherapy may be an optimal choice. However, $40 \%$ to $75 \%$ of the patients developed drug resistance or disease progression soon after treatment, resulting in treatment failure (22). Therefore, finding effective drugs is the key to treating this fatal disease.

The traditional chemotherapy regimen for advanced EC is cisplatin combined with fluorouracil. In recent years, chemotherapeutic drugs such as paclitaxel, docetaxel, irinotecan, and nedaplatin have also been widely used in clinical practice, with an objective response rate (ORR) of $15 \%$ to $45 \%$ (23). However, the duration of response (DOR) of first-line regimens was only 4 to 6 months, with a median survival time (MST) of 10 to 12 months (23). Most ECs in China are esophageal squamous cell carcinoma (ESCC), which account for $90 \%$ of all cases of EC worldwide (24). ESCC differs from adenocarcinoma, in its epidemiology, pathogenesis, biology, and prognosis (25). When patients receive therapy, most cancer cells are usually eradicated, but subpopulations of cells that are resistant to the treatment may remain alive and lead to recurrence. This phenomenon is mainly due to the existence of CSCs (26).

CCSs are a heterogeneous cell population with unlimited proliferation, self-renewal, multi-directional differentiation, pluripotency, and resistance to conventional radiotherapy and chemotherapy (6). Unlike non-CSCs, CSCs are static or dormant, so are resistant to treatment such as

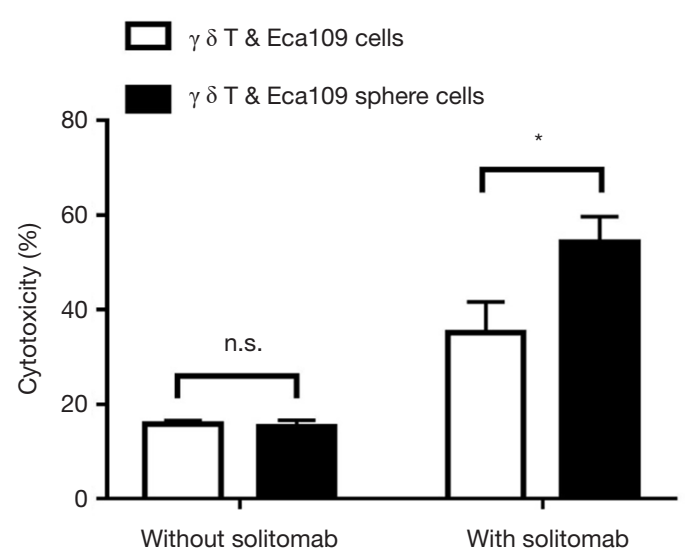

Figure 5 Analysis of cytotoxicity of solitomab combined with $\gamma \delta$ T cells on wild-type Eca109 cells and Eca109 cell spheres. *, There was no significant difference in the cytotoxicity of $\gamma \delta$ T cells to wild type Eca109 cells and Eca109 cell sphere cells without solitomab $(\mathrm{P}>0.05) . \gamma \delta \mathrm{T}$ cells significantly enhanced the cytotoxicity of both groups of cells and the cytotoxicity of $\gamma$ $\delta$ T cells to Eca109 cell sphere cells was significantly higher than that of wild type Eca109 cells with solitomab $(\mathrm{P}<0.05)$. n.s., not significant.

radiotherapy and chemotherapy, and this can lead to tumor recurrence or metastasis when CSCs re-enter the cell cycle $(27,28)$. At present, CSCs have been successfully identified and isolated from different tumors $(29,30)$ and studies have reported that the existence of EC stem cells is the main reason for radiotherapy and chemotherapy resistance $(4,5)$. Therefore, in this study, we used serum-free suspension culture to induce the formation of Eca109 cell spheres.

Serum-free suspension culture has been widely used in the isolation and culture of various CSCs $(31,32)$. Zhang et al. (33) have reported the application of cell spheres culture to isolate and enrich potential CSC subpopulations from EC Eca109 cell. Wang et al. (34) successfully obtained passaging cell spheres from KYSE150 and TE1 EC cell lines by serum-free suspension culture. They found that the expression level of the stem cell gene in sphere cells was significantly increased, and the sphere cells were more resistant to radiotherapy than parent cells. This proved there were more CSCs in cell spheres than in parent cells. In the present study, EC Eca109 cells were cultured in serum-free RPMI1640 medium containing B27 supplement for 3 days to form suspended cell spheres. The cell spheres were collected and blown into single cell suspension and then passaged. On the 12th day of culture, first generation 
cell spheres that had a strong ability for proliferation and self-renewal were formed. Flow cytometry analysis showed that Eca109 cell spheres were smaller in size and had less cytoplasmic granules than wild-type Eca109 cells.

CSCs exhibit multidrug resistance to chemotherapeutic drugs. Liao et al. (31) cultured spherical cells from human ovarian cancer cell lines and primary ovarian cancer with stronger malignant potential in growth, invasion, scratch recovery, clone survival, migration, and chemotherapy resistance, which demonstrated that spheroid cells had the characteristics of CSCs. In addition, Sun et al. (32) found that cultured EC OE-19 cell spheres had more obvious drug resistance compared with non-sphere OE-19 cells. Nab-PTX is a new chemotherapeutic drug composed of human serum albumin and paclitaxel complex using nanotechnology. Studies have reported that Nab-PTX combined with cisplatin for EC is effective and tolerable (35). Therefore, in our study, different concentrations of NabPTX and cisplatin were used on wild-type Eca109 cells and Eca109 sphere cells, respectively. After 24 hours, the growth of cells in both groups was inhibited and the proportion of surviving cells decreased with increasing drug concentration. CCK-8 assay showed that both group of cells died under the different concentrations of NabPTX and cisplatin, and the viability of Eca109 cell sphere cells was significantly higher than that of wild-type Eca109 cells. This suggests Eca109 cell spheres have stronger drug resistance than wild-type Eca109 cells and may contain a higher proportion of EC stem cells.

EpCAM, also known as CD326, was first found in human colorectal cancer cells with mouse monoclonal antibody 17-1A by Hilary Koprowski in 1979. Therefore, it is considered as a tumor-associated antigen in human colorectal cancer tissue (36). It is a glycosylated type I membrane protein with a molecular weight of $30-40 \mathrm{kDa}$ and consists of a extracellular domain (EpEX) which determines the stability of EpCAM on the cell surface, a single spanning transmembrane domain (TM), and a short cytoplasmic domain (EpIC) (37).

Typically, EpCAM is expressed in the first developmental stages of highly proliferating cells such as progenitors and embryonic stem cells, and its expression is decreased in differentiated cells. In adults, EpCAM expression is restricted to epithelial tissues, specifically at the basolateral cell membranes. However, its expression increases under pathological conditions such as inflammation and cancer, which is of great significance to the development of cancer (38). EpCAM is considered as one of the most important biomarkers of cancer and only expresses in tumors of epithelial origin, such as pancreatic, small intestine, breast, ovarian, esophageal, and gastric cancer, but not in ectoderm or mesoderm tumors (39). Research on the expression of EpCAM in renal cell carcinoma showed that EpCAM was expressed in clear cell carcinoma, oncocytoma and papillary carcinoma, with an expression ratio of $36.3 \%, 78.3 \%$ and $81.3 \%$, respectively (40). Sun et al. (32) found that there was a high expression of EpCAM in EC tissue and EC derived cell line OE-19EC tissue. In the present study, we used flow cytometry to detect the expression level of EpCAM in wildtype Eca109 cells and Eca109 cell sphere cells. The results showed that EpCAM expression was observed in both groups, which confirmed that EpCAM may be a biomarker for EC.

Many studies have reported that the frequency and range of EpCAM expression in some CSCs, such as pancreatic, colon, breast, and prostate cancer are extensive and frequent than non-CSCs $(14,20,21)$. Sun (32) found that the expression level of EpCAM of drug-resistant OE19 cell spheres induced by ACF (adriamycin, cisplatin, and fluorouracil) was significantly higher than that of nonspherical OE-19 cells. They suggested ACF induced the high expression of EpCAM, reflecting the occurrence of esophageal adenocarcinoma (EAC) and the selection of a drug-resistant stem cell subpopulation. Terris et al. (12) reported that EpCAM-positive hepatocellular carcinoma (HCC) cells were tumor initiation cells with stem or progenitor cell characteristics and EpCAM is a new marker of HCC stem cells. The growth and invasiveness of HCC were determined by EpCAM-positive cells, which provides a new therapeutic target for the treatment of HCC. In our study, we found that the expression level of Eca109 cell spheres was significantly higher than that of wild-type Eca109 cells by flow cytometry, suggesting that EpCAM was expressed more frequently in EC stem cells, which verified that Eca109 cell spheres may contain a high proportion of EC stem cells. It has been reported that the mechanism of the relationship between the enhancement of malignant potential of epithelial cells and increased expression lever of EpCAM was related to cell cycle signaling and increased activity of proto-oncogenes (41). EpCAM was also associated with cellular signals through the Wnt pathway to enhance the characteristics of CSCs that is related to chemotherapy resistance (42). Therefore, the exploration of therapeutic drugs targeting EpCAM for $\mathrm{EC}$ is expected to improve tumor resistance to treatment.

In recent years, antibody-based therapy has become 
a promising means of tumor immunotherapy. With the development of bispecific antibody (bsAb) engineering, bsAb has become a new choice for cancer immunotherapy. BsAb has the unique ability to bind two or three different epitopes at the same time, which can be divided into bispecific $\mathrm{T}$ cell binding antibody (bispecific $\mathrm{T}$ cell engager, BiTE) and trispecific antibody (TrAb). Therefore, they can act as connectors between tumor cells and immune effector cells [neutrophils, T cells, macrophages, or natural killer (NK) cells], thus activating immune cells and stimulating the host's innate and acquired immune response, leading to tumor cell lysis and enabling tumor cells to overcome tumor immune escape mechanism and killing tumor cells (43).

Solitomab is an EpCAM/CD3 bispecific single-chain antibody construct which can bind to tumor cells expressing EpCAM and $\mathrm{T}$ cells to activate $\mathrm{T}$ cells to kill tumor cells. Its anti-tumor mechanism is to activate $T$ cells to induce tumor cell apoptosis by releasing perforin and granzyme to dissolve tumor cells $(15,16)$. It was also reported that solitomab could induce the polyclonal activation of $\mathrm{CD}^{+}$ and $\mathrm{CD} 8^{+} \mathrm{T}$ cells, which was characterized by the reexpression of CD69 and CD25 and the secretion of IFN $-\gamma$, TNF- $\alpha$ and IL-2, IL-4, and IL-10. In addition, solitomab also strongly influences $\mathrm{CD}^{+} \mathrm{T}$ cells to eliminate tumor cells and $\mathrm{CD}^{+} \mathrm{T}$ cells may also kill tumor cells due to the upregulation of granzyme B expression (15). Other results have also shown that a large proportion of $\mathrm{CD} 8^{+}$ and $\mathrm{CD}^{+} \mathrm{T}$ cells in peripheral blood were involved in the killing of tumor cells by solitomab. Cioffi et al. (18) showed that established cell lines of pancreatic cancer were more responsive to solitomab-activated $\mathrm{T}$ cells than that of primary cells, and primary cells also showed a dose- and time-dependent response to treatment with the solitomab. Both in vivo and in vitro experiments showed that solitomab could effectively kill the highly tumorigenic pancreatic CSC subpopulation. Ferrari et al. (44) co-cultured ovarian sarcoma cells with high expression of EpCAM with solitomab and $\mathrm{T}$ cells and the cytotoxicity of $\mathrm{T}$ cells to tumor cells was significantly enhanced. After the tumor cells expressing EpCAM in pleural effusion were incubated with autologous pleural effusion, the proliferation of $\mathrm{CD} 4^{+}$ and $\mathrm{CD} 8^{+} \mathrm{T}$ cells was significantly enhanced, the $\mathrm{T}$ cell activation markers increased significantly, and the viability of tumor cells decreased significantly. Solitomab also had a significant inhibitory effect on uterine cancer, uterine sarcoma, and colon cancer cell lines $(15,17,18)$. The safety and efficacy of solitomab were evaluated in phase I clinical trials of patients with lung cancer and gastrointestinal tumors, and showed higher anti-tumor activity in animal models (15). However, the cytotoxicity of solitomab on EC cells in vivo and in vitro has not been reported. In the present study, co-culture experiment showed that there was no significant difference in the cytotoxicity of $\gamma \delta \mathrm{T}$ cells to wild type Eca109 cells or Eca109 cell sphere cells without solitomab, and after its addition, $\gamma \delta \mathrm{T}$ cells could significantly enhance the cytotoxicity of both groups of cells. The cytotoxicity of $\gamma \delta$ T cells to Eca109 cell spheres was significantly higher than that of wild type Eca109 cells, which showed that $\gamma \delta \mathrm{T}$ cells combined with solitomab had stronger cytotoxicity on the subsets of tumor cells with high expression of EpCAM. EpCAM may be a potential molecular target of immunotherapy for EC, and solitomab may become an effective immunotherapeutic drug for EC resistant to chemotherapy.

Together, the Eca109 cell spheres obtained by serumfree suspension culture had stronger characteristics of CSCs such as proliferation, multidrug resistance, and high expression level of EpCAM. Our experimental results confirm that Eca109 cell spheres may contain a high proportion of EC stem cells. It is inferred that EpCAM may be a potential molecular target of immunotherapy for EC, and solitomab may become an effective immunotherapeutic drug for EC resistant to chemotherapy. This study provides an experimental basis for finding effective drugs to treat EC resistant to chemotherapy and improving the survival of patients with EC, and offers a theoretical basis for exploring the synergistic anti-tumor effect of bispecific antibodies combined with immune cells.

\section{Acknowledgments}

Funding: None.

\section{Footnote}

Reporting Checklist: The authors have completed the MDAR reporting checklist. Available at http://dx.doi.org/10.21037/ jtd-21-442

Data Sharing Statement: Available at http://dx.doi. org/10.21037/jtd-21-442

Conflicts of Interest: All authors have completed the ICMJE uniform disclosure form (available at http://dx.doi. org/10.21037/jtd-21-442). The authors have no conflicts of interest to declare. 
Ethical Statement: The authors are accountable for all aspects of the work in ensuring that questions related to the accuracy or integrity of any part of the work are appropriately investigated and resolved.

Open Access Statement: This is an Open Access article distributed in accordance with the Creative Commons Attribution-NonCommercial-NoDerivs 4.0 International License (CC BY-NC-ND 4.0), which permits the noncommercial replication and distribution of the article with the strict proviso that no changes or edits are made and the original work is properly cited (including links to both the formal publication through the relevant DOI and the license). See: https://creativecommons.org/licenses/by-nc-nd/4.0/.

\section{References}

1. Bray F, Ferlay J, Soerjomataram I, et al. Global cancer statistics 2018: GLOBOCAN estimates of incidence and mortality worldwide for 36 cancers in 185 countries. CA Cancer J Clin 2018;68:394-424.

2. Lao-Sirieix P, Fitzgerald RC. Screening for oesophageal cancer. Nat Rev Clin Oncol 2012;9:278-87.

3. Pennathur A, Gibson MK, Jobe BA, et al. Oesophageal carcinoma. Lancet 2013;381:400-12.

4. Wang D, Plukker JTM, Coppes RP. Cancer stem cells with increased metastatic potential as a therapeutic target for esophageal cancer. Semin Cancer Biol 2017;44:60-6.

5. Wu Q, Wu Z, Bao C, et al. Cancer stem cells in esophageal squamous cell cancer. Oncol Lett 2019;18:5022-32.

6. Kharkar PS. Cancer stem cell (CSC) inhibitors: a review of recent patents (2012-2015). Expert Opin Ther Pat 2017;27:753-61.

7. Hemmati HD, Nakano I, Lazareff JA, et al. Cancerous stem cells can arise from pediatric brain tumors. Proc Natl Acad Sci U S A 2003;100:15178-83.

8. Singh SK, Clarke ID, Terasaki M, et al. Identification of a cancer stem cell in human brain tumors. Cancer Res 2003;63:5821-8.

9. Tayama S, Motohara T, Narantuya D, et al. The Impact of EpCAM Expression on Response to Chemotherapy and Clinical Outcomes in Patients With Epithelial Ovarian Cancer. Oncotarget 2017;8:44312-25.

10. Wu F, Zhu J, Mao Y, et al. Associations between the Epithelial-Mesenchymal Transition Phenotypes of Circulating Tumor Cells and the Clinicopathological Features of Patients with Colorectal Cancer. Dis Markers 2017;2017:9474532.
11. Ni J, Cozzi P, Beretov J, et al. Epithelial cell adhesion molecule (EpCAM) is involved in prostate cancer chemotherapy/radiotherapy response in vivo. BMC Cancer 2018;18:1092.

12. Terris B, Cavard C, Perret C. EpCAM, a new marker for cancer stem cells in hepatocellular carcinoma. J Hepatol 2010;52:280-1.

13. Heiler S, Wang Z, Zoller M. Pancreatic cancer stem cell markers and exosomes - the incentive push. World J Gastroenterol 2016;22:5971-6007.

14. Gires O, Klein CA, Baeuerle PA. On the abundance of EpCAM on cancer stem cells. Nat Rev Cancer 2009;9:143.

15. Brischwein K, Schlereth B, Guller B, et al. MT110: A Novel Bispecific Single-Chain Antibody Construct With High Efficacy in Eradicating Established Tumors. Mol Immunol 2006;43:1129-43.

16. Eyvazi S, Farajnia S, Dastmalchi S, et al. Antibody Based EpCAM Targeted Therapy of Cancer, Review and Update. Current Cancer Drug Targets 2018;18:857-68.

17. Bellone S, Black J, English DP, et al. Solitomab, an EpCAM/CD3 bispecific antibody construct (BiTE), is highly active against primary uterine serous papillary carcinoma cell lines in vitro. Am J Obstet Gynecol 2016;214:99.e1-8.

18. Cioffi M, Dorado J, Baeuerle PA, et al. EpCAM/CD3Bispecific T-cell Engaging Antibody MT110 Eliminates Primary Human Pancreatic Cancer Stem Cells. Clin Cancer Res 2012;18:465-74.

19. Yazdanifar M, Barbarito G, Bertaina A, et al. gammadelta T Cells: The Ideal Tool for Cancer Immunotherapy. Cells 2020;9:1305.

20. Yahyazadeh Mashhadi SM, Kazemimanesh M, Arashkia A, et al. Shedding light on the EpCAM: An overview. J Cell Physiol 2019;234:12569-80.

21. Visvader JE, Lindeman GJ. Cancer stem cells in solid tumours: accumulating evidence and unresolved questions. Nat Rev Cancer 2008;8:755-68.

22. Farinella E, Safar A, Nasser HA, et al. Salvage esophagectomy after failure of definitive radiochemotherapy for esophageal cancer. J Surg Oncol 2016;114:833-7.

23. Ku GY. Systemic therapy for esophageal cancer: chemotherapy. Chin Clin Oncol 2017;6:49.

24. Torre LA, Bray F, Siegel RL, et al. Global cancer statistics, 2012. CA Cancer J Clin 2015;65:87-108.

25. Rustgi AK, Ingelfinger JR, El-Serag HB. Esophageal Carcinoma. N Engl J Med 2014;371:2499-509.

26. Maugeri-Saccà M, Vigneri $\mathrm{P}, \mathrm{De}$ Maria R. Cancer 
Stem Cells and Chemosensitivity. Clin Cancer Res 2011;17:4942-7.

27. Chen W, Dong J, Haiech J, et al. Cancer Stem Cell Quiescence and Plasticity as Major Challenges in Cancer Therapy. Stem Cells Int 2016;2016:1740936.

28. Takeishi S, Nakayama KI. To wake up cancer stem cells, or to let them sleep, that is the question. Cancer Sci 2016;107:875-81.

29. Collins AT, Berry PA, Hyde C, et al. Prospective Identification of Tumorigenic Prostate Cancer Stem Cells. Cancer Res 2005;65:10946-51.

30. Vermeulen L, Todaro M, de Sousa Mello F, et al. Singlecell cloning of colon cancer stem cells reveals a multilineage differentiation capacity Proc Natl Acad Sci U S A 2008;105:13427-32.

31. Liao J, Qian F, Tchabo N, et al. Ovarian Cancer Spheroid Cells With Stem Cell-Like Properties Contribute to Tumor Generation, Metastasis and Chemotherapy Resistance Through Hypoxia-Resistant Metabolism. PLoS One 2014;9:e84941.

32. Sun X, Martin RCG, Zheng Q, et al. Drug-induced expression of EpCAM contributes to therapy resistance in esophageal adenocarcinoma. Cell Oncol (Dordr) 2018;41:651-62.

33. Zhang G, Ma L, Xie YK, et al. Esophageal cancer tumorspheres involve cancer stem-like populations with elevated aldehyde dehydrogenase enzymatic activity. Mol Med Rep 2012;6:519-24.

34. Wang JL, Yu JP, Sun ZQ, et al. Radiobiological Characteristics of Cancer Stem Cells From Esophageal Cancer Cell Lines. World J Gastroenterol 2014;20:18296-305.

35. Shi Y, Qin R, Wang ZK, et al. Nanoparticle albumin-

Cite this article as: Yu L, Guo QM, Wang Y, Xu Y, Liu L, Zhang XT. EpCAM expression in esophageal cancer and its correlation with immunotherapy of solitomab. J Thorac Dis 2021;13(4):2404-2413. doi: 10.21037/jtd-21-442 bound paclitaxel combined with cisplatin as the firstline treatment for metastatic esophageal squamous cell carcinoma. Onco Targets Ther 2013;6:585-91.

36. Koprowski H, Steplewski Z, Mitchell K, et al. Colorectal carcinoma antigens detected by hybridoma antibodies. Somatic Cell Genet 1979;5:957-71.

37. Imrich $S$, Hachmeister M, Gires O. EpCAM and its potential role in tumor-initiating cells. Cell Adh Migr 2012;6:30-8.

38. Baeuerle PA, Gires O. EpCAM (CD326) finding its role in cancer. British Journal of Cancer 2007;96:417-23.

39. Armstrong A, Eck SL. EpCAM-a new therapeutic target for an old cancer antigen. Cancer Biol Ther 2003;2:320-6.

40. Zimpfer A, Maruschke M, Rehn S, et al. Prognostic and diagnostic implications of epithelial cell adhesion/ activating molecule (EpCAM) expression in renal tumours: a retrospective clinicopathological study of 948 cases using tissue microarrays. BJU Int 2014;114:296-302.

41. Munz M, Baeuerle PA, Gires O. The emerging role of EpCAM in cancer and stem cell signaling. Cancer Res 2009;69:5627-9.

42. Sumithra B, Saxena U, Das AB. Alternative splicing within the Wnt signaling pathway: role in cancer development. Cell Oncol (Dordr) 2016;39:1-13.

43. Grandjenette C, Dicato M, Diederich M. Bispecific antibodies: an innovative arsenal to hunt, grab and destroy cancer cells. Curr Pharm Biotechnol 2015;16:670-83.

44. Ferrari F, Bellone S, Black J, et al. Solitomab, an EpCAM/ CD3 bispecific antibody construct (BiTE(R)), is highly active against primary uterine and ovarian carcinosarcoma cell lines in vitro. J Exp Clin Cancer Res 2015;34:123.

(English Language Editor: B. Draper) 